\title{
Effect of dietary oils on peripheral neuropathy- related endpoints in dietary obese rats
}

This article was published in the following Dove Press journal:

Diabetes, Metabolic Syndrome and Obesity:Targets and Therapy

\section{Lawrence Coppey' \\ Eric Davidson' \\ Hanna Shevalye' \\ Michael E Torres' \\ Mark A Yorek ${ }^{1-4}$}

'Department of Internal Medicine, University of lowa, lowa City, IA, USA; ${ }^{2}$ Department of Veterans Affairs lowa City Health Care System, lowa City, IA, USA; ${ }^{3}$ Department of Veterans Affairs, Veterans Affairs Center for the Prevention and Treatment of Visual Loss, lowa City, IA, USA; ${ }^{4}$ Fraternal Order of Eagles Diabetes Research Center, University of lowa, lowa City, IA, USA
Correspondence: Mark A Yorek Building 4I, Room 127, 60I Highway 6 West, Veteran Affairs Medical Center, lowa City, IA 52246, USA

Tel + I 3 I 9338 058I ext 7696

Fax +l 3193397162

Email mark-yorek@uiowa.edu
Purpose: This study aimed to determine the effect of dietary oils (olive, safflower, evening primrose, flaxseed, or menhaden) enriched in different mono unsaturated fatty acids or polyunsaturated fatty acids on peripheral neuropathies in diet-induced obese Sprague-Dawley rats. Materials and methods: Rats at 12 weeks of age were fed a high-fat diet ( $45 \% \mathrm{kcal})$ for 16 weeks. Afterward, the rats were fed diets with $50 \%$ of the kilocalories of fat derived from lard replaced by the different dietary oils. In addition, a control group fed a standard diet (4\% kcal fat) and a high fat fed group ( $45 \% \mathrm{kcal})$ were maintained. The treatment period was 32 weeks. The endpoints evaluated included motor and sensory nerve conduction velocity, thermal sensitivity, innervation of sensory nerves in the cornea and skin, and vascular relaxation by epineurial arterioles.

Results: Menhaden oil provided the greatest benefit for improving peripheral nerve damage caused by dietary obesity. Similar results were obtained when we examined acetylcholinemediated vascular relaxation of epineurial arterioles of the sciatic nerve. Enriching the diets with fatty acids derived from the other oils provided minimal to partial improvements.

Conclusion: These studies suggest that omega-3 polyunsaturated fatty acids derived from fish oil could be an effective treatment for neural and vascular complications associated with obesity. Keywords: peripheral neuropathy, fish oil, omega-3 polyunsaturated fatty acids, omega-6 polyunsaturated fatty acids, vascular reactivity, nerve conduction velocity

\section{Introduction}

Peripheral neuropathy affects about $50 \%$ of patients with diabetes as well as $30 \%$ of obese patients with insulin resistance. ${ }^{1}$ This suggests factors other than hyperglycemia contribute to peripheral neuropathy. Since symptoms of peripheral neuropathy include pain, numbness, paresthesia, and ulcerations in the extremities, finding treatment is vital. $^{2}$ Lipids have been shown to play an important role in complications associated with metabolic syndrome and type 2 diabetes. ${ }^{3}$ However, little information is available regarding the effect of different classes of fatty acids on peripheral neuropathy. Our earlier studies suggest that long-chain omega-3 (n-3) polyunsaturated fatty acids, commonly found in fish oils (primarily eicosapentaenoic and docosahexaenoic acids), enable effective treatment for vascular complications and peripheral neuropathy associated with obesity and type 2 diabetes. ${ }^{4-6}$ Omega- 3 fatty acids have been shown to have broad health benefits, such as lowering blood triglycerides, improving cardiovascular disease, and decreasing the risk of certain types of cancers. ${ }^{7}$ However, the potential role of omega-3 polyunsaturated fatty acids as well as the other classes of monounsaturated and polyunsaturated fatty acids on peripheral neuropathy has been understudied. 
To gain a better understanding of the role of dietary lipids in peripheral neuropathy in an animal model of prediabetes, rats were fed a high-fat diet for 16 weeks followed by 32 weeks of diet enriched in oleic acid (olive oil; 18:1, n-9), linoleic acid (safflower oil; 18:2, n-6), $\gamma$-linolenic acid (evening primrose oil; 18:3, n-6), $\alpha$-linolenic acid (flaxseed oil; 18:3, n-3), or eicosapentaenoic/docosahexaenoic acids (menhaden oil; 20:5 and 22:6, n-3).

\section{Materials and methods}

\section{Animals, diets, and experimental design}

Male Sprague-Dawley (Harlan Sprague Dawley, Indianapolis, IN, USA) rats 10-11 weeks of age were housed in a certified animal care facility and food (\#7001; Harlan Teklad, Madison, WI, USA) and water were provided ad libitum. All institutional and National Institutes of Health (NIH) guidelines for the use of animals were followed. These studies were approved by the University of Iowa Animal Care and Use Committee (\#5071450). At 12 weeks of age, rats were divided into seven groups. One group was designated as the control group and remained on standard diet, which contained $4.25 \mathrm{gm} \%$ as fat. The other six groups of rats were placed on a high-fat diet (D12451; Research Diets, New Brunswick, NJ, USA). The high-fat diet contained $24 \mathrm{gm} \%$ fat, $24 \mathrm{gm} \%$ protein, and $41 \mathrm{gm} \%$ carbohydrate. The primary source of the increased fat content in the diet was lard. Rats were maintained on these diets for 16 weeks. Afterward, five of the six groups of rats on the high-fat diet were placed on high-fat diets with $50 \% \mathrm{kcal}$ of fat derived from lard replaced with olive oil (D16030902), safflower oil (D16030903), flaxseed oil (D16030904), evening primrose oil (D16030905), or menhaden oil (D16021504). These diets were prepared by Research Diets and maintained for 32 weeks. The fatty acid composition of each of these diets is presented in Table S1. Two groups designated as control and diet-induced obese (DIO) rats were continued on their respective diets until the end of the study.

\section{Glucose clearance}

Glucose tolerance was determined 1 week before the terminal vascular and nerve studies by injecting rats with a saline solution containing $2 \mathrm{~g} / \mathrm{kg}$ glucose, intraperitoneally, after an overnight fast. ${ }^{4}$ Rats were briefly anesthetized with isoflurane and the glucose solution was injected. Immediately prior to the glucose injection and at $15,30,45,60$, 120,180 , and 240 minutes blood samples (one drop) from the tip of the tail were taken to measure circulating glucose levels using Aviva Accu-Chek glucose oxidase reagent strips (Roche Diabetes Care, Inc., Indianapolis, IN, USA).

\section{Endpoints related to nerve and vascular reactivity}

A variety of endpoints related to neural function were determined employing methodology common to our laboratory and detailed information can be found in the references. ${ }^{4,6,8}$ These endpoints included thermal nociceptive latency of the hindpaws and cornea sensitivity in unanesthetized rats. Motor and sensory nerve conduction velocity and density of corneal nerves were determined in rats anesthetized with sodium pentobarbital $(50 \mathrm{mg} / \mathrm{kg}$, intraperitoneally; Abbott Laboratories, Abbott Park, IL, USA). Following these procedures, the anesthetized rats were euthanized by exsanguination and tissue harvested to determine intraepidermal nerve fiber density of the hindpaw and vascular reactivity of epineurial arterioles of the sciatic nerve to acetylcholine.

\section{Fatty acid composition}

Fatty acid composition of the diets, serum, and liver was measured after the lipid fraction was extracted using chloroform/methanol, followed by transesterification in $14 \%$ boron trifluoride in methanol and extraction of the fatty acid methyl esters into heptane. The fatty acids were then separated by gas-liquid chromatography. ${ }^{9}$ Individual fatty acids peaks as \% of the total fatty acids present were identified by comparison to known fatty acid standards.

\section{Physiological markers}

Nonfasting blood glucose was determined with Aviva AccuChek strips. Serum was collected for determining levels of free fatty acid, triglyceride, and free cholesterol using commercial kits from Hoffman-La Roche (Basel, Switzerland), Sigma-Aldrich Co (St Louis, MO, USA), and BioVision (Milpitas, CA, USA), respectively. Serum was also used to determine thiobarbituric acid reactive substances (TBARS) as previously described. ${ }^{8}$

\section{Data analysis}

Results are presented as mean \pm standard error of mean. Comparisons between the groups were conducted using one-way analysis of variance and Bonferroni posttest comparison (Prism software; GraphPad Software Inc, La Jolla, CA, USA). Concentration response curves for acetylcholine were compared using a two-way repeated measures analysis of variance with autoregressive covariance structure using Proc 
Mixed program of SAS Institute Inc (Cary, NC, USA). ${ }^{4,6,8}$ A $p$-value $<0.05$ was considered to be significant.

\section{Results Weight and blood glucose}

At the beginning of the study, all rats weighed the same (Table 1). After 16 weeks of the high-fat diet, the high-fatfed rats weighed significantly more than the control rats. At the end of the study, all rats continued to gain weight and supplementing the high-fat diet with oils enriched in monounsaturated fatty acid or polyunsaturated fatty acid for the final 32 weeks of the 48-week study period did not significantly impact the final weight gain. High-fat-fed rats and rats fed diets supplemented with oils enriched in monounsaturated fatty or polyunsaturated fatty acid consumed about the same amount of food and significantly less than control rats. Nonfasting blood glucose was determined with Aviva Accu-Chek strips at the end of the study and all rats were normoglycemic. Serum lipid and thiobarbituric acid substances levels were also determined (Table 1). Serum-free fatty acid levels were significantly elevated in high-fat-fed rats, and high-fat-fed rats supplemented with olive oil, safflower oil, flaxseed oil, and evening primrose oil compared to control rats. Supplementing high-fat-fed rats with menhaden oil lowered serum-free fatty acid levels. Serum triglyceride levels were significantly increased in high-fat-fed rats supplemented with olive oil or evening primrose oil compared to control rats. Serum TBARS were significantly increased in high-fat-fed rats compared to control rats. Supplementing high-fat-fed rats with olive oil, safflower oil, and flaxseed oil lowered TBARS levels. However, supplementing high-fatfed rats with evening primrose oil or menhaden oil corrected TBARS levels. Figure S1 demonstrates that enriching the diet of DIO rats with evening primrose oil or flaxseed oil improved glucose utilization. In contrast, enriching their diets with olive oil, safflower oil, or menhaden oil was far less beneficial.

\section{Fatty acid composition of serum and liver}

Tables S2 and S3 present data for the fatty acid composition of serum and liver following the dietary intervention of dietinduced obese rats with oils enriched in oleic acid (olive oil), linoleic acid (safflower oil), $\gamma$-linolenic acid (evening primrose oil), $\alpha$-linolenic acid (flaxseed oil), or eicosapentaenoic and docosahexaenoic acids (menhaden oil). In serum, the primary fatty acid enriched in each of the oils was significantly increased compared to control and/or high-fat-fed rats. With the exception of olive oil and evening primrose oil, the fatty acid(s) enrichment occurring in liver from safflower oil, flaxseed oil, and menhaden oil was those fatty acids that were enriched in these oils and were significant compared to liver from DIO rats. Rats fed a diet enriched with evening primrose oil had significantly higher amounts of arachidonic acid in liver compared to high-fat-fed rats. In liver, the unsaturation index was significantly increased in DIO rats treated with diets enriched in olive oil, flaxseed oil, or menhaden oil; however, serum was unchanged (Table S4). An increase in the omega-6 to omega-3 fatty acid ratio has been linked to increased inflammatory stress in different diseases, whereas a decrease in this ratio corresponds with a decrease in inflammatory stress. ${ }^{10,11}$ Data in Table S5 demonstrate that the omega- 6 to omega- 3 fatty acid ratio in liver is increased in DIO rats treated with dietary safflower oil or evening primrose oil compared to control and high-fat-fed rats and decreased in DIO rats treated with flaxseed oil or menhaden oil. The omega-6 to omega-3 fatty acid ratio in

Table I Effect of dietary oils on weight gain, diet consumption, nonfasting blood glucose, and serum-free fatty acid, triglyceride, cholesterol, and TBARS in DIO Sprague-Dawley rats

\begin{tabular}{|c|c|c|c|c|c|c|c|}
\hline Determination & $\begin{array}{l}\text { Control } \\
\text { (9) }\end{array}$ & $\begin{array}{l}\text { DIO } \\
\text { (II) }\end{array}$ & $\begin{array}{l}\text { DIO+ } \\
\text { olive oil } \\
(9)\end{array}$ & $\begin{array}{l}\text { DIO+ } \\
\text { safflower oil } \\
\text { (9) }\end{array}$ & $\begin{array}{l}\text { DIO+ } \\
\text { flaxseed oil } \\
\text { (9) }\end{array}$ & $\begin{array}{l}\text { DIO+evening } \\
\text { primrose oil } \\
\text { (8) }\end{array}$ & $\begin{array}{l}\text { DIO+ } \\
\text { menhaden oil } \\
(9)\end{array}$ \\
\hline Start weight (g) & $35 I \pm 4$ & $336 \pm 5$ & $343 \pm 5$ & $344 \pm 4$ & $34 I \pm 3$ & $352 \pm 4$ & $334 \pm 5$ \\
\hline Weight at treatment (g) & $465 \pm I I$ & $530 \pm 8 *$ & $536 \pm 9 *$ & $533 \pm 10 *$ & $544 \pm 9 *$ & $536 \pm 8 *$ & $54 I \pm 9 *$ \\
\hline End weight $(g)$ & $530 \pm 18$ & $647 \pm 27^{*}$ & $682 \pm 21 *$ & $693 \pm 13^{*}$ & $706 \pm 14 *$ & $668 \pm 12^{*}$ & $645 \pm 14^{*}$ \\
\hline Diet consumed (g/kg body wt/day) & $43 \pm 1$ & $28 \pm I^{*}$ & $29 \pm 1 *$ & $29 \pm 1 *$ & $30 \pm I^{1}$ & $28 \pm 1 *$ & $29 \pm 1 *$ \\
\hline Blood glucose $(\mathrm{mg} / \mathrm{dL})$ & $161 \pm 12$ & $162 \pm 14$ & $162 \pm 4$ & $158 \pm 7$ & $155 \pm 6$ & $169 \pm 7$ & $147 \pm 7$ \\
\hline Free fatty acid (mmol/L) & $0.23 \pm 0.06$ & $0.48 \pm 0.09 *$ & $0.42 \pm 0.05^{*}$ & $0.55 \pm 0.12^{*}$ & $0.60 \pm 0.08 *$ & $0.53 \pm 0.13^{*}$ & $0.32 \pm 0.05$ \\
\hline Triglyceride $(\mathrm{mg} / \mathrm{mL})$ & $36 \pm 3$ & $48 \pm 6$ & $75 \pm 8 *$ & $48 \pm 7$ & $60 \pm 8$ & $56 \pm 3 *$ & $35 \pm 6$ \\
\hline Cholesterol (mg/mL) & $1.7 \pm 0.2$ & $2.0 \pm 0.1$ & $1.9 \pm 0.1$ & $1.8 \pm 0.2$ & $2.2 \pm 0.4$ & $2.0 \pm 0.2$ & $1.2 \pm 0.4$ \\
\hline TBARS (mg/mL) & $0.59 \pm 0.09$ & $0.88 \pm 0.05^{*}$ & $0.69 \pm 0.06$ & $0.69 \pm 0.06$ & $0.78 \pm 0.06$ & $0.47 \pm 0.15$ & $0.57 \pm 0.11$ \\
\hline
\end{tabular}

Notes: Data are presented as the mean \pm standard error of mean. $* p<0.05$ compared to control. Parentheses indicate the number of experimental animals.

Abbreviations: TBARS, thiobarbituric acid substances; DIO, diet-induced obese; wt, weight. 
serum was also decreased in diet-induced obese rats treated with flaxseed oil or menhaden oil.

\section{Neural and vascular endpoints}

Chronic obesity has been associated with neural impairment and endothelial dysfunction independent of hyperglycemia. ${ }^{12,13}$ Figure 1 shows that a diet of $45 \%$ kcal fat derived primarily from lard for 48 weeks results in slowing of motor and sensory nerve conduction velocity. Replacing $50 \% \mathrm{kcal}$ of the fat derived from lard with olive oil for the latter 32 weeks did not improve nerve conduction velocities. Replacing $50 \% \mathrm{kcal}$ of the fat with safflower oil, flaxseed oil, or evening primrose oil provided some benefit primarily in motor nerve conduction velocity. In contrast, when the high-fat diet was enriched with menhaden oil both motor and sensory nerve conduction velocities were significantly improved.

Another common neuropathic endpoint is the determination of intraepidermal nerve fiber density and thermal sensitivity. Figure 2 demonstrates that in DIO rats, there is a significant decrease in nerve fibers in the skin and latent response to a thermal stimulus. Treating DIO rats with olive oil, flaxseed oil, or evening primrose oil had no effect on these two endpoints. Treatment with safflower oil significantly improved intraepidermal nerve fiber density but not thermal sensitivity. In contrast, treatment with menhaden oil significantly improved both intraepidermal nerve fiber density and thermal nociception.

Recently, examination of corneal nerve fiber density of the subepithelial layer and cornea sensitivity has been promoted as a surrogate marker for progression of diabetic neuropathy in human subjects..$^{14}$ It has also been shown that loss of corneal nerves occurs in subjects with impaired glucose tolerance..$^{15}$ Figure 3 shows that diet-induced obesity causes a decrease in corneal nerves and abnormal cornea sensitivity. Treating DIO rats with olive oil or safflower oil did not correct these abnormalities. Treatment with flaxseed oil or evening primrose oil partially improved cornea nerve density and function. However, treatment of DIO rats with menhaden oil completely restored both cornea nerve density and sensitivity.

We have previously demonstrated that the vascular reactivity of epineurial arterioles, blood vessels that provide circulation to the sciatic nerve, to acetylcholine is decreased prior to the slowing of nerve conduction velocity in diabetic and DIO rats. ${ }^{16,17}$ Figure 4 confirms that acetylcholine-mediated vascular relaxation is impaired in DIO rats. Treating DIO rats with olive oil, safflower oil, flaxseed oil, or evening primrose

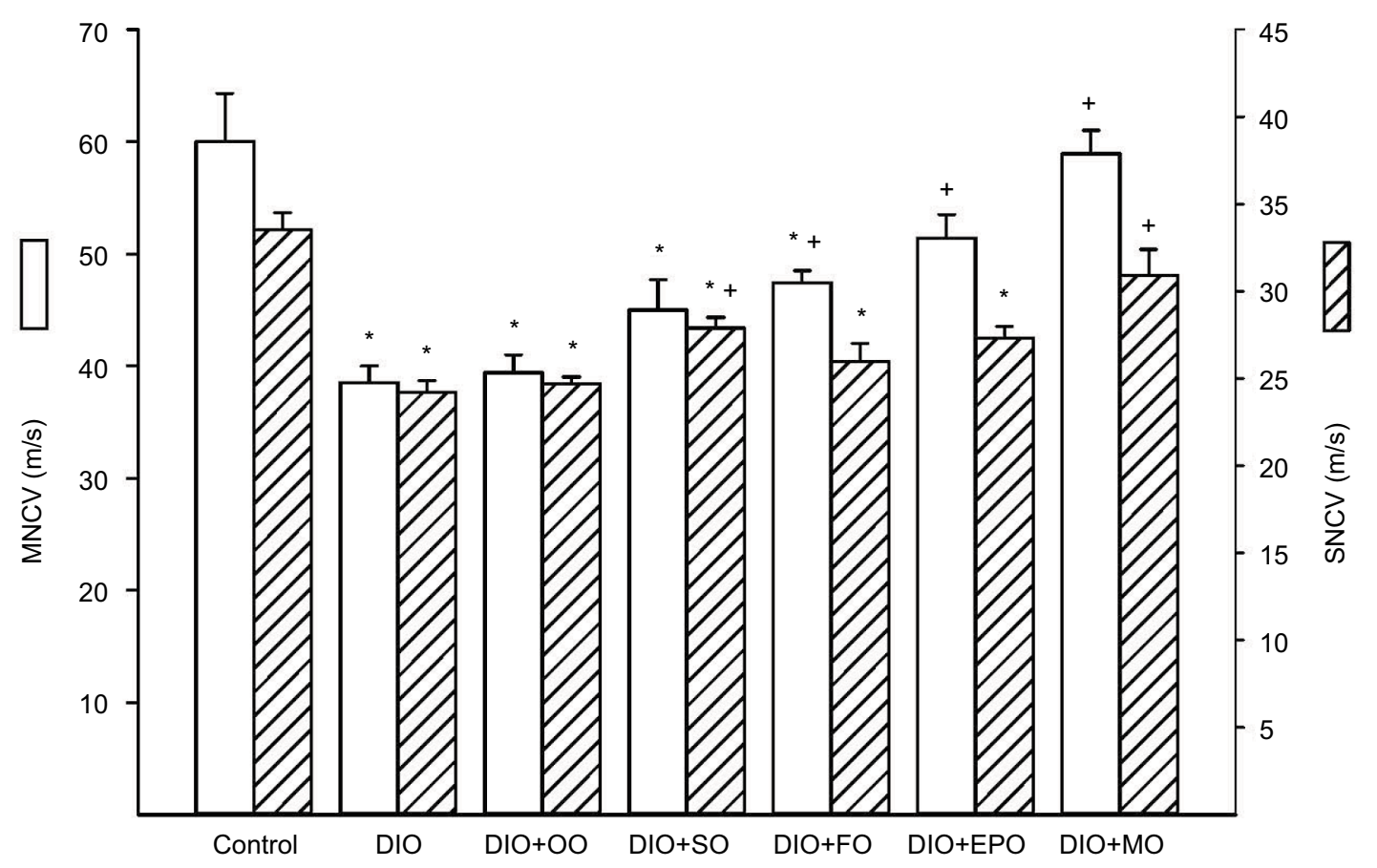

Figure I Effect of dietary oils on motor and sensory nerve conduction velocity in DIO Sprague-Dawley rats.

Notes: Motor and sensory nerve conduction velocities were examined as described in the "Materials and methods" section. Data are presented as the mean \pm standard error of mean in $\mathrm{m} / \mathrm{s}$. The number of rats in each group was the same as shown in Table I. ${ }^{*} p<0.05$ compared to control rats; ${ }^{+} p<0.05$ compared to DIO rats.

Abbreviations: MNCV, motor nerve conduction velocity; SNCV, sensory nerve conduction velocity; DIO, diet-induced obese; OO, olive oil; SO, safflower oil; FO, flaxseed oil; EPO, evening primrose oil; MO, menhaden oil. 


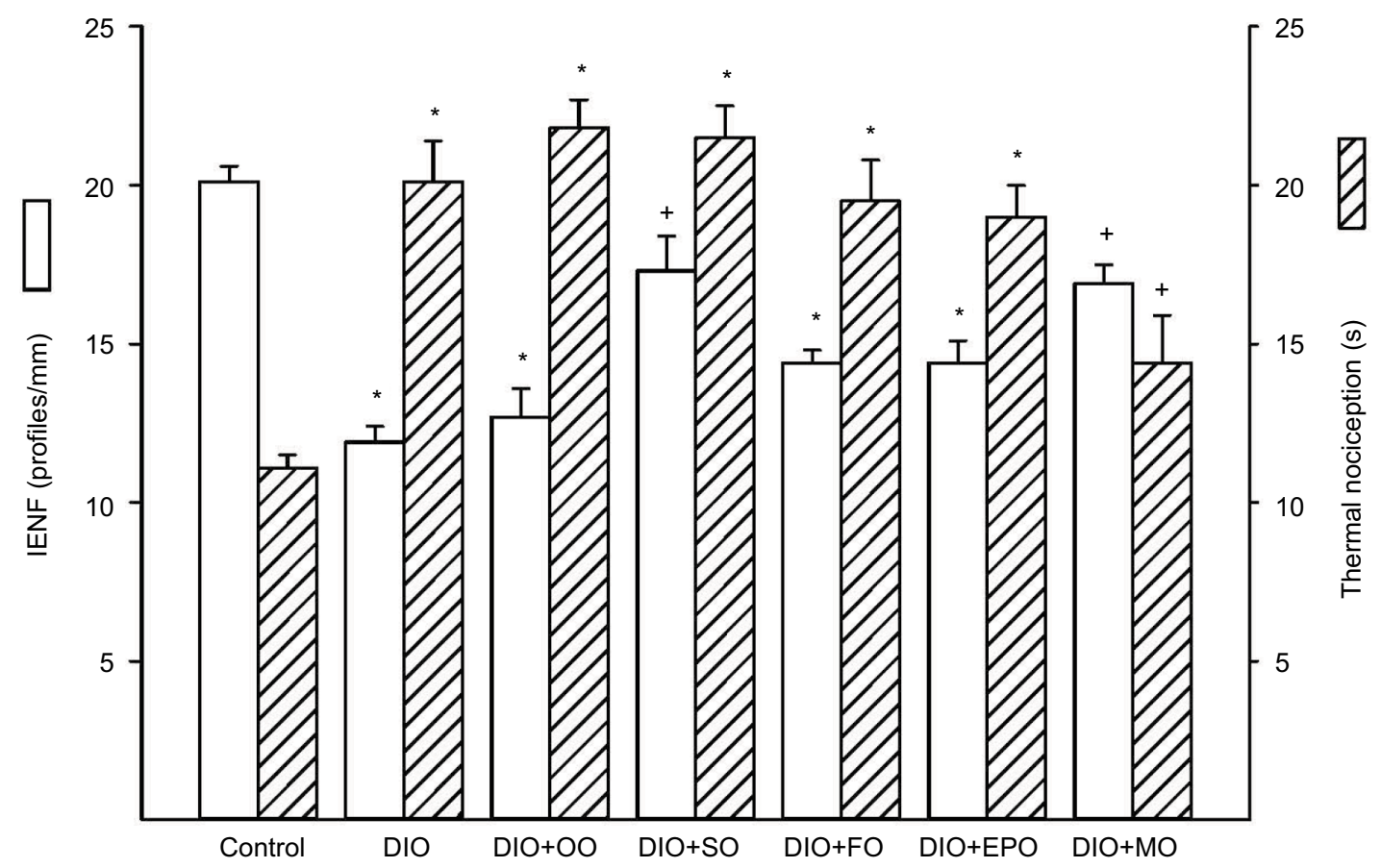

Figure 2 Effect of dietary oils on intraepidermal nerve fiber density and thermal nociception in DIO Sprague-Dawley rats.

Notes: Intraepidermal nerve fiber density and thermal nociception were examined as described in the "Materials and methods" section. Data are presented as the mean \pm standard error of mean for intraepidermal nerve fiber profiles per $\mathrm{mm}$ and thermal nociception in seconds. The number of rats in each group was the same as shown in Table I. ${ }^{*} p<0.05$ compared to control rats; ${ }^{+} p<0.05$ compared to DIO rats.

Abbreviations: IENF, intraepidermal nerve fiber density; DIO, diet-induced obese; OO, olive oil; SO, safflower oil; FO, flaxseed oil; EPO, evening primrose oil; MO, menhaden oil.

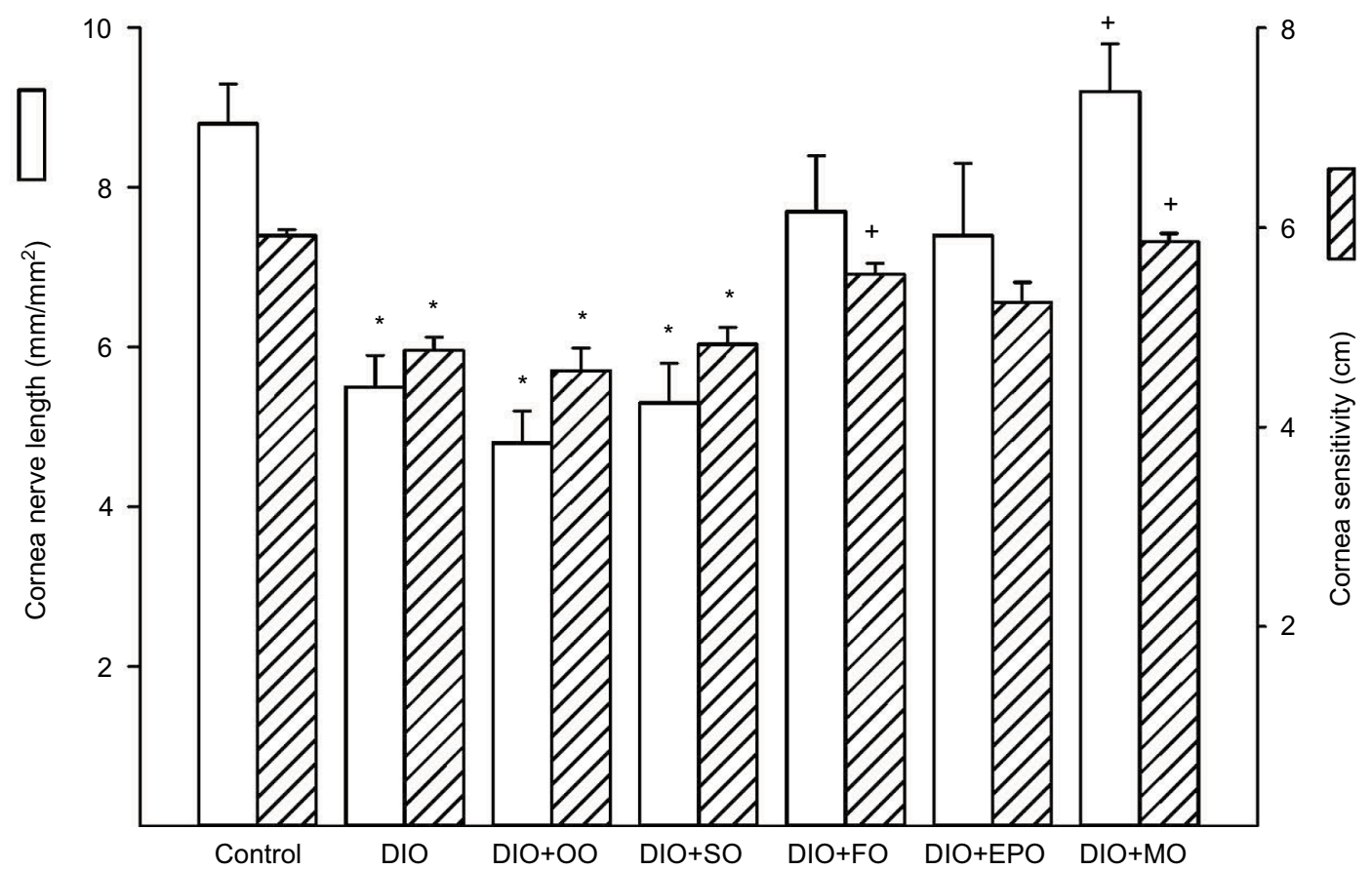

Figure 3 Effect of dietary oils on cornea nerve fiber length and cornea sensitivity in DIO Sprague-Dawley rats.

Notes: Innervation of the subepithelial layer of the cornea and corneal sensitivity were determined by using corneal confocal microscopy and Cochet-Bonnet filament esthesiometer, respectively, as described in the "Materials and methods" section. The number of rats in each group was the same as shown in Table I. Data are presented as the mean \pm standard error of mean for innervation of the cornea in $\mathrm{mm} / \mathrm{mm}^{2}$ and for corneal sensitivity in centimeters. ${ }^{*} p<0.05$ compared to control rats; ${ }^{+} p<0.05$ compared to DIO rats.

Abbreviations: DIO, diet-induced obese; OO, olive oil; SO, safflower oil; FO, flaxseed oil; EPO, evening primrose oil; MO, menhaden oil. 


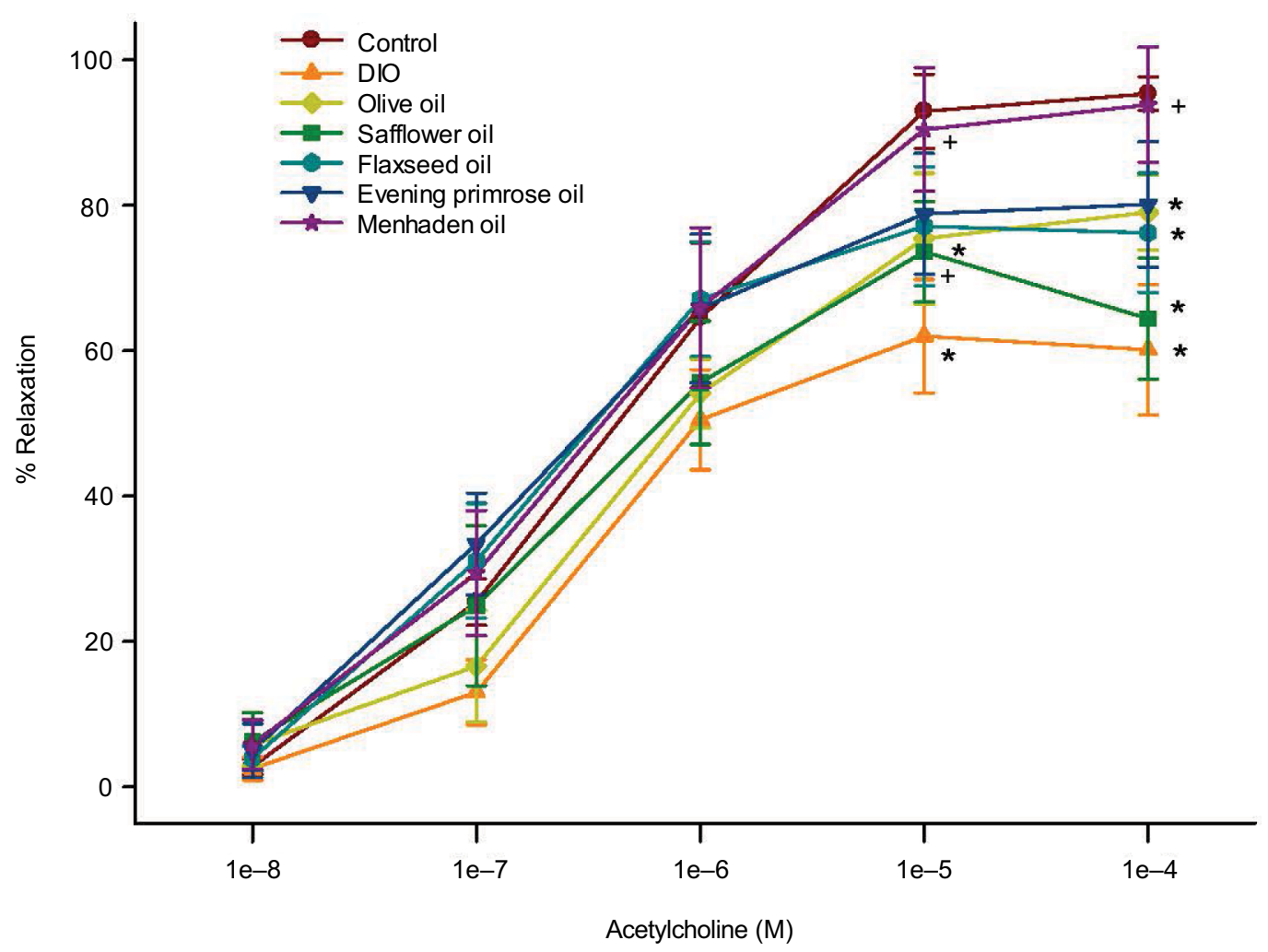

Figure 4 Effect of dietary oils on vascular relaxation by acetylcholine in epineurial arterioles of the sciatic nerve in DIO Sprague-Dawley rats.

Notes: Pressurized arterioles ( $40 \mathrm{~mm} \mathrm{Hg}$ and ranging from 60 to $100 \mu \mathrm{m}$ luminal diameters) were constricted with phenylephrine (30\%-50\%) and incremental doses of acetylcholine were added to the bathing solution while recording steady state vessel diameter. The number of rats in each group was the same as shown in Table I. Data are presented as the mean of $\%$ relaxation \pm standard error of mean. ${ }^{*} p<0.05$ compared to control rats; ${ }^{+} p<0.05$ compared to DIO rats.

Abbreviation: DIO, diet-induced obese.

oil partially improved vascular relaxation to acetylcholine. However, treating DIO rats with menhaden oil totally restored vascular relaxation to acetylcholine.

\section{Discussion}

We have previously reported that obese Zucker rats and DIO Sprague-Dawley rats develop vascular and neural impairment independent of hyperglycemia. ${ }^{18,19}$ The source of lipid in many high-fat diets used to induce obesity is lard, which contains a large amount of saturated fatty acids. It is unknown what effect other classes of monounsaturated fatty or polyunsaturated fatty acid may have on vascular and neural defects in DIO rats. To address this question, we fed DIO rats diets for 32 weeks with $50 \% \mathrm{kcal}$ of fat derived from lard replaced with olive oil (enriched in oleic acid, 18:1, n-9 monounsaturated fatty acid), safflower oil (enriched in linoleic acid, 18:2, n-6 polyunsaturated fatty acid), evening primrose oil (enriched in $\gamma$-linolenic acid, 18:3, n-6 polyunsaturated fatty acid), flaxseed oil (enriched in $\alpha$-linolenic acid, 18:3, n-3 polyunsaturated fatty acid), or menhaden oil (enriched in eicosapentaenoic and docosahexaenoic fatty acid, 20:5 and 22:6, n-3 polyunsaturated fatty acid). It is important to stress that the study design was an intervention-like protocol and only $50 \% \mathrm{kcal}$ of fat derived from lard was substituted with the desired oils. Prior to treating obese rats with the different classes of mono and polyunsaturated fatty acids, Sprague-Dawley rats were fed a high-fat diet for 16 weeks. At the time of treatment, these rats were obese and had impaired vascular and neural function as previously reported. ${ }^{17}$

After 48 weeks, rats fed a high-fat diet with fatty acids derived primarily from lard had extensive vascular and neural damage including slowing of motor and sensory nerve conduction velocities, loss of nerve fibers in the skin and cornea, and decreased thermal sensitivity in the hindpaw and mechanical sensitivity of the cornea. In previous studies, rats fed a high-fat diet for a shorter period of time had slowing of sensory nerve conduction velocity but motor nerve conduction velocity was not impaired. ${ }^{17,19}$ Thus, it seems that a longer duration of obesity causes a progressive loss of neural function.

When the obese rats were fed a diet enriched with oleic acid derived from olive oil, there was minimal to no effect on glucose utilization and neural function and a moderate 
but not significant improvement in vascular reactivity of epineurial arterioles to acetylcholine. The fatty acid composition of serum and liver was mostly unchanged in DIO rats treated with olive oil-enriched diet compared to DIO rats. Our finding that olive oil provides minimal benefit for vascular and neural function in dietary obese rats differs from other studies. It has been shown that increased consumption of olive oil improves endothelial function, lowers blood pressure, and reduces lipotoxicity associated with obesity in animal and human subjects, which is thought to be due to a reduction in oxidative and inflammatory stress. $^{20-22}$ The lack of an effect in our studies could be due to our starting the diet well after obesity-induced vascular and neural dysfunction had developed. The starting point, delivery, and duration of treatment also vary greatly in published studies.

Treating DIO rats with the omega-6 polyunsaturated fatty acid, linoleic acid, derived from safflower oil, like olive oil, provided minimal to no benefit in vascular and neural function with only sensory nerve conduction velocity being partially improved. It has been reported that the increased dietary level of safflower oil causes a reduction in glucose disposal in skeletal muscle that is due to decreased glycolytic flux and increased free fatty acid/ ketone oxidation. ${ }^{23}$

Treating DIO rats with the omega- 6 polyunsaturated fatty acid, $\gamma$-linolenic acid, derived from evening primrose oil, or the omega-3 polyunsaturated fatty acid, $\alpha$-linolenic acid, derived from flaxseed oil, was more effective than safflower oil in improving glucose clearance, vascular reactivity of epineurial arterioles to acetylcholine, cornea nerve fiber length and cornea sensitivity, and motor nerve conduction velocity. This occurred even though the content of $\gamma$-linolenic acid in the evening primrose oil-enriched diet increased only from $2.3 \% \pm 0.6 \%$ in the high-fat diet to $5.4 \% \pm 0.8 \%$. In contrast, the flaxseed oil-enriched diet contained $31.0 \% \pm 3.3 \% \alpha$-linolenic acid. It has been widely reported that $\gamma$-linolenic acid improves vascular and neural function including endoneurial blood flow of the sciatic nerve in diabetic rats. ${ }^{24-26} \gamma$-Linolenic acid has been reported to have anti-inflammatory properties and improves abnormal lipid and thromboxane metabolism in diabetes. ${ }^{27,28} \alpha$-Linolenic acid has been reported to improve glucose tolerance in obese rats and insulin sensitivity/ glycemic control in human subjects with prediabetes or type 2 diabetes. ${ }^{29-31}$ It has also been reported to prevent endothelial dysfunction in type 2 diabetic rats by enhancing endothelial nitric oxide synthase activity and reducing oxidative/nitrative stress. ${ }^{32}$

Increasing the concentration of omega- 3 fatty acids reduces the omega- 6 to omega- 3 ratio, thereby reducing inflammatory stress. ${ }^{33}$ However, in obesity and diabetes, the activities of delta 5 and delta 6 desaturases are decreased. ${ }^{34-38}$ Decrease in the activity of these enzymes reduces the elongation and desaturation of linolenic fatty acids of both omega- 6 and omega- 3 classes. The omega- 3 polyunsaturated fatty acids, eicosapentaenoic and docosahexaenoic acids, are precursors for $\mathrm{E}$ and $\mathrm{D}$ classes of resolvins that have anti-inflammatory properties. ${ }^{39,40}$ In our study, there was a significant increase in eicosapentaenoic acid but no change in docosahexaenoic acid levels in the liver of DIO rats treated with flaxseed oil. When DIO rats were treated with menhaden oil, there were greater increases in both eicosapentaenoic and docosahexaenoic acids. Thus, treating obese rats with menhaden oil theoretically would bypass the decrease in delta 5 and delta 6 desaturase activities, thereby potentially increasing the availability of eicosapentaenoic and docosahexaenoic acids for the production of $\mathrm{E}$ and $\mathrm{D}$ classes of resolvins, respectively. When DIO rats were treated with menhaden oil, vascular reactivity to acetylcholine was fully restored, motor and sensory nerve conduction velocities were corrected to control values, and sensory nerve density in the skin and cornea as well as sensitivity were significantly improved. The greater benefit observed in vascular and neural function in DIO rats treated with menhaden oil versus the other classes of fatty acids, including $\alpha$-linolenic acid derived from flaxseed oil, could be due to the greater decrease in the omega- 6 to omega- 3 ratio in the liver and serum and increased formation of resolvins.

\section{Conclusion}

Our studies have shown that treating DIO rats, after vascular and nerve pathology have developed, with oils enriched in oleic acid and linoleic acid did not improve vascular or neural function. We found that treating these rats with either $\gamma$-linolenic acid or $\alpha$-linolenic acid, derived from evening primrose oil or flaxseed oil, respectively, provided moderate benefit. In contrast, treating these rats with menhaden oil enriched in eicosapentaenoic and docosahexaenoic acids provided significant improvement in vascular and neural function. Increasing the dietary intake of fish oils may be a potential treatment strategy for vascular and neural complications associated with obesity and prediabetes. 


\section{Acknowledgments}

Research reported in this publication was supported by the Department of Veterans Affairs, Veterans Health Administration, Office of Research and Development Merit award (RX000889-05), by a National Institute of Diabetes and Digestive and Kidney Diseases of the NIH award (DK107339), and by a National Heart, Lung, and Blood Institute of the NIH award (T35HL007485). The abstract of this paper was presented at two meetings as a poster presentation with interim findings; first, at the annual NEURODIAB 2017 meeting held on September 9-11 in Coimbra, Portugal and second at the University of Iowa Medical Student Research Conference, held on September 13-15, 2017. The poster's abstract was for the NEURODIAB meeting published on the NEURODIAB2017 website (http://www.neurodiab2017. com/AbstractBook.aspx). For the University of Iowa Medical Student Research Conference, the abstract was published on the Carver College of Medicine website (https://medicine. uiowa.edu/md/sites/medicine.uiowa.edu.md/files/2017\%20 Abstract\%20Book.pdf).

\section{Author contributions}

LC, EC, HS, and MET assisted in design, performance of the studies, and data analysis as well as preparation of the manuscript. MET was also responsible for animal care. ED was involved in the preparation of figures. MAY was responsible for the overall projects including design of the studies, overseeing the performance of the studies, data analysis, and writing the manuscript. All authors contributed toward data analysis, drafting and revising the paper and agree to be accountable for all aspects of the work.

\section{Disclosure}

The authors report no conflicts of interest in this work.

\section{References}

1. Cortez M, Singleton JR, Smith AG. Glucose intolerance, metabolic syndrome, and neuropathy. Handb Clin Neurol. 2014;126:109-122.

2. Yorek MA. Is fish oil a potential treatment for diabetic peripheral neuropathy? Curr Diabetes Rev. 2017;13:1-11.

3. Vincent AM, Callaghan BC, Smith AL, Feldman EL. Diabetic neuropathy: cellular mechanisms as therapeutic targets. Nat Rev Neurol. 2011;7:573-583.

4. Coppey LJ, Holmes A, Davidson EP, Yorek MA. Partial replacement with menhaden oil improves peripheral neuropathy in high fat fed low dose streptozotocin type 2 diabetic rat. J Nutr Metab. 2012;2012:950517.

5. Lamping KG, Nuno DW, Coppey LJ, et al. Modification of high saturated fat diet with n-3 polyunsaturated fat improves glucose intolerance and vascular dysfunction. Diabetes Obes Metab. 2013;15:144-152.

6. Coppey LJ, Davidson EP, Obrosov A, Yorek MA. Enriching the diet with menhaden oil improves peripheral neuropathy in streptozotocin-induced type 1 diabetic rats. J Neurophysiol. 2015;113:701-708.
7. Wang X, Chan CB. n-3 Polyunsaturated fatty acids and insulin secretion. J Endocrinol. 2015;224:R97-R106.

8. Davidson EP, Coppey LJ, Shevalye H, Obrosov A, Kardon RH, Yorek MA. Impaired corneal sensation and nerve loss in a type 2 rat model of chronic diabetes is reversible with combination therapy of menhaden oil, $\alpha$-lipoic acid, and enalapril. Cornea. 2017;36:725-731.

9. Yorek MA, Bohnker RR, Dudley DD, Spector AA. Comparative utilization of n-3 polyunsaturated fatty acids by cultured human Y-79 retinoblastoma cells. Biochim Biophys Acta. 1984;795:277-285.

10. Valenzuela R, Videla LA. The importance of the long-chain polyunsaturated fatty acid n-6/n-3 ratio in development of non-alcoholic fatty liver associated with obesity. Food Funct. 2011;2:644-648.

11. Wall R, Ross RP, Fitzgerald GF, Stanton C. Fatty acids from fish: the anti-inflammatory potential of long-chain omega-3 fatty acids. Nutr Rev. 2010;68:280-289.

12. Ghosh A, Gao L, Thakur A, Siu PM, Lai CWK. Role of free fatty acids in endothelial dysfunction. J Biomed Sci. 2017;24:50.

13. Stino AM, Smith AG. Peripheral neuropathy in prediabetes and the metabolic syndrome. J Diabetes Investig. 2017;8:646-655.

14. Edwards K, Pritchard N, Vagenas D, Russell A, Malik RA, Efron N. Utility of corneal confocal microscopy for assessing mild diabetic neuropathy: baseline findings of the LANDMark study. Clin Exp Optom. 2012;95:348-354.

15. Azmi S, Ferdousi M, Petropoulos IN, et al. Corneal confocal microscopy identifies small-fiber neuropathy in subjects with impaired glucose tolerance who develop type 2 diabetes. Diabetes Care. 2015;38:1502-1508.

16. Coppey LJ, Davidson EP, Dunlap JA, Lund DD, Yorek MA. Slowing of motor nerve conduction velocity in streptozotocin-induced diabetic rats is preceded by impaired vasodilation in arterioles that overlie the sciatic nerve. Int J Exp Diabetes Res. 2000;1:131-143.

17. Davidson EP, Coppey LJ, Calcutt NA, Oltman CL, Yorek MA. Dietinduced obesity in Sprague-Dawley rats causes microvascular and neural dysfunction. Diabetes Metab Res Rev. 2010;26:306-318.

18. Oltman CL, Coppey LJ, Gellett JS, Davidson EP, Lund DD, Yorek MA. Progression of vascular and neural dysfunction in sciatic nerves of Zucker diabetic fatty (ZDF) and Zucker rats. Am J Physiol Endocrinol Metab. 2005;289:E113-E122.

19. Davidson EP, Coppey LJ, Dake B, Yorek MA. Effect of treatment of Sprague Dawley rats with AVE7688, enalapril, or candoxatril on dietinduced obesity. J Obes. 2011;2011:686952.

20. Davis CR, Hodgson JM, Woodman R, Bryan J, Wilson C, Murphy KJ. A Mediterranean diet lowers blood pressure and improves endothelial function: results from the MedLey randomized intervention trial. $\mathrm{Am}$ J Clin Nutr. 2017;105:1305-1313.

21. Elias S, Wisam S, Luai A, Massad B, Nimer A. Lipotoxicity in obesity: benefit of olive oil. Adv Exp Med Biol. 2017;960:607-617.

22. Schwingshackl L, Christoph M, Hoffmann G. Effects of olive oil on markers of inflammatory and endothelial function - a systematic review and meta-analysis. Nutrients. 2015;7:7651-7675.

23. Jucker BM, Cline GW, Barucci N, Shulman GI. Differential effects of safflower oil versus fish oil feeding on insulin-stimulated glycogen synthesis, glycolysis, and pyruvate dehydrogenase flux in skeletal muscle: a 13C nuclear magnetic resonance study. Diabetes. 1999;48: 134-140.

24. Jack AM, Keegan A, Cotter MA, Cameron NE. Effects of diabetes and evening primrose oil treatment on responses of aorta, corpus cavernosum and mesenteric vasculature in rats. Life Sci. 2002;71:1863-1877.

25. Omawari N, Dewhurst M, Vo P, Mahmood S, Stevens E, Tomlinson DR. Deficient nitric oxide responsible for reduced nerve blood flow in diabetic rats: effects of L-NAME, L-arginine, sodium nitroprusside and evening primrose oil. Br J Pharmacol. 1996;118:186-190.

26. Cameron NE, Cotter MA. Effects of evening primrose oil treatment on sciatic nerve blood flow and endoneurial oxygen tension in streptozotocin-diabetic rats. Acta Diabetol. 1994;31:220-225.

27. Kapoor R, Huang YS. Gamma linolenic acid: an antiinflammatory omega-6 fatty acid. Curr Pharm Biotechnol. 2006;7:531-534. 
28. Takahashi R, Inoue J, Ito H, Hibino H. Evening primrose oil and fish oil in non-insulin-dependent-diabetes. Prostaglandins Leukot Essent Fatty Acids. 1993;49:569-571.

29. Matravadia S, Zabielski P, Chabowski A, Mutch DM, Holloway GP. LA and ALA prevent glucose intolerance in obese male rats without reducing reactive lipid content, but cause tissue-specific changes in fatty acid composition. Am J Physiol Regul Integr Comp Physiol. 2016;310: R619-R630.

30. Gomes PM, Hollanda-Miranda WR, Beraldo RA, et al. Supplementation of $\alpha$-linolenic acid improves serum adiponectin levels and insulin sensitivity in patients with type 2 diabetes. Nutrition. 2015;31: 853-857.

31. Hutchins AM, Brown BD, Cunnane SC, Domitrovich SG, Adams ER, Bobowiec CE. Daily flaxseed consumption improves glycemic control in obese men and women with pre-diabetes: a randomized study. Nutr Res. 2013;33:367-375.

32. Zhang W, Fu F, Tie R, et al. Alpha-linolenic acid intake prevents endothelial dysfunction in high-fat diet-fed streptozotocin rats and underlying mechanisms. Vasa. 2013;42:421-428.

33. Yang LG, Song ZX, Yin H, et al. Low n-6/n-3 PUFA ratio improves lipid metabolism, inflammation, oxidative stress and endothelial function in rats using plant oils as n-3 fatty acid source. Lipids. 2016;51:49-59.
34. Wolters M, Schlenz H, Bornhorst C, et al. Desaturase activity is associated with weight status and metabolic risk markers in young children. J Clin Endocrinol Metab. 2015;100:3760-3769.

35. Araya J, Rodrigo R, Pettinelli P, Araya AV, Poniachik J, Videla LA. Decreased liver fatty acid delta- 6 and delta-5 desaturase activity in obese patients. Obesity. 2010;18:1460-1463.

36. Warensjo E, Rosell M, Hellenius ML, Vessby B, De Faire U, Riserus U. Associations between estimated fatty acid desaturase activities in serum lipids and adipose tissue in humans: links to obesity and insulin resistance. Lipids Health Dis. 2009;8:37.

37. Tosi F, Sartori F, Guarini P, Olivieri O, Martinelli N. Delta-5 and delta-6 desaturases: crucial enzymes in polyunsaturated fatty acid-related pathways with pleiotropic influences in health and disease. Adv Exp Med Biol. 2014;824:61-81.

38. Kroger J, Schulze MB. Recent insights into the relation of $\Delta 5$ desaturase and $\Delta 6$ desaturase activity to the development of type 2 diabetes. $C u r r$ Opin Lipidol. 2012;23:4-10.

39. Chiang N, Serhan CN. Structural elucidation and physiologic functions of specialized pro-resolving mediators and their receptors. Mol Aspects Med. 2017;58:114-129.

40. Serhan CN, Chiang N, Dalli J, Levy BD. Lipid mediators in the resolution of inflammation. Cold Spring Harb Perspect Biol. 2014;7:a016311. 


\section{Supplementary materials}

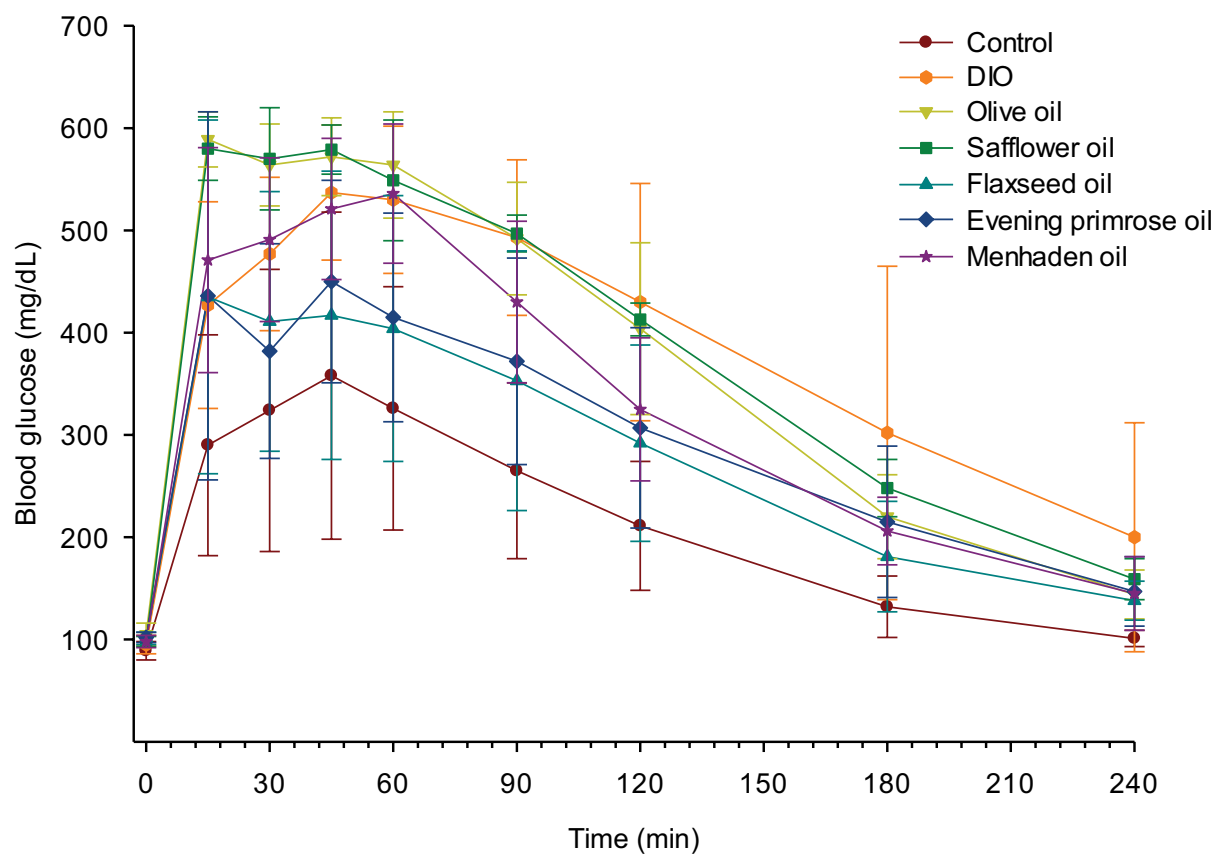

Figure SI Effect of dietary oils on glucose clearance in DIO Sprague-Dawley rats.

Notes: Rats were fed a standard or high-fat diet for 16 weeks. Afterward, $50 \%$ kcal derived from lard in the high-fat diet was replaced with olive oil, safflower oil, flaxseed oil, evening primrose oil, or menhaden oil. Rats were maintained on these diets as well as on standard and high-fat diets for an additional 32 weeks. Glucose utilization was determined as described in the "Materials and methods" section. Data are presented as the mean \pm standard error of mean in mg/dL. The area under the curve was significantly different $(p<0.05)$ for high-fat-fed rats and high-fat-fed rats treated with olive oil, safflower oil, or menhaden oil versus control. The number of rats in each group was the same as shown in Table I.

Abbreviation: DIO, diet-induced obese.

Table SI Fatty acid \% composition of diets

\begin{tabular}{|c|c|c|c|c|c|c|c|c|}
\hline Diet & $16: 0$ & 18:0 & $18: 1$ & $18: 2$ & $18: 3(n-6)$ & $18: 3(n-3)$ & $20: 5$ & $22: 6$ \\
\hline Control & $21 \pm 3.0$ & $7.3 \pm 1.2$ & $30.4 \pm 4.0$ & $31.7 \pm 5.1$ & $2.8 \pm 0.6$ & $<1$ & $<1$ & $<1$ \\
\hline High fat & $16.8 \pm 2.2$ & $10.8 \pm 1.3$ & $41.2 \pm 5.6$ & $26.9 \pm 2.8$ & $2.3 \pm 0.3$ & $<1$ & $<1$ & $<1$ \\
\hline Olive oil & $14.9 \pm 2.4$ & $5.4 \pm 0.7$ & $49.7 \pm 6.3$ & $21.3 \pm 3.0$ & $2.0 \pm 0.1$ & $<1$ & $<1$ & $<1$ \\
\hline Safflower oil & $12.3 \pm 0.7 *$ & $6.0 \pm 0.5$ & $24.9 \pm 3.4$ & $54.3 \pm 4.3^{*,+}$ & $<1^{*,+}$ & $<1$ & $<1$ & $<1$ \\
\hline Flaxseed oil & $11.1 \pm 1.0^{*++}$ & $6.2 \pm 1.0$ & $27.0 \pm 2.8$ & $23.6 \pm 3.4$ & $<1^{*,+}$ & $31.0 \pm 3.3^{*,+}$ & $<1$ & $<1$ \\
\hline Evening primrose oil & $11.6 \pm 1.7^{*,+}$ & $6.0 \pm 0.7$ & $20.7 \pm 2.4$ & $52.5 \pm 4.0^{*,+}$ & $5.4 \pm 0.8$ & $<1$ & $<1$ & $<1$ \\
\hline Menhaden oil & $18.7 \pm 0.9$ & $7.1 \pm 0.6$ & $22.6 \pm 2.6$ & $16.0 \pm 0.9$ & $<1^{*,+}$ & $2.0 \pm 0.1$ & $9.5 \pm 1.0 *,+$ & $6.9 \pm 0.4^{*,+}$ \\
\hline
\end{tabular}

Notes: Data are presented as the mean of three determinations \pm standard error of mean. ${ }^{*} p<0.05$ compared to control; ${ }^{+} p<0.05$ compared to high fat.

Table S2 Effect of dietary lipids on fatty acid \% composition of serum measured by gas chromatography

\begin{tabular}{|c|c|c|c|c|c|c|c|c|c|}
\hline Diet & $16: 0$ & $18: 0$ & |8:1 & $18: 2$ & $18: 3(n-6)$ & $18: 3(n-3)$ & $20: 4$ & $20: 5$ & $22: 6$ \\
\hline Control (9) & $23.1 \pm 1.7$ & $17.2 \pm 1.2$ & $10.2 \pm 0.5$ & $13.8 \pm 0.7$ & $0.6 \pm 0.5$ & $0.4 \pm 0.1$ & $18.9 \pm 2.0$ & $0.7 \pm 0.2$ & $3.1 \pm 0.5$ \\
\hline High fat (II) & $19.4 \pm 0.6$ & $21.5 \pm 0.6$ & $8.4 \pm 0.5$ & $16.4 \pm 0.5$ & $0.3 \pm 0.1$ & $0.4 \pm 0.1$ & $21.0 \pm 0.9$ & $0.4 \pm 0.1$ & $3.2 \pm 0.3$ \\
\hline High fat+olive oil (8) & $19.1 \pm 0.7^{*}$ & $20.9 \pm 0.6$ & $11.1 \pm 0.6^{+}$ & $13.4 \pm 1.0$ & $0.1 \pm 0.0$ & $0.3 \pm 0.0$ & $21.3 \pm 1.0$ & $0.5 \pm 0.1$ & $4.7 \pm 0.7$ \\
\hline High fat+safflower oil (9) & $18.3 \pm 0.5^{*}$ & $22.3 \pm 0.7$ & $5.9 \pm 0.3^{*,+}$ & $17.7 \pm 0.8^{*}$ & $0.3 \pm 0.1$ & $0.8 \pm 0.4$ & $2 \mathrm{I} .4 \pm \mathrm{I} . \mathrm{I}$ & $0.6 \pm 0.3$ & $3.0 \pm 0.3$ \\
\hline High fat+flaxseed oil (8) & $19.3 \pm 0.5^{*}$ & $20.9 \pm 0.9$ & $7.8 \pm 1.1$ & $17.3 \pm 0.6$ & $0.2 \pm 0.1$ & $2.5 \pm 0.8^{*,+}$ & $15.5 \pm 0.1^{+}$ & $1.9 \pm 0.1^{+}$ & $4.9 \pm 0.4$ \\
\hline High fat+evening primrose oil (7) & $17.9 \pm 0.7^{*}$ & $22.1 \pm 1.2$ & $5.1 \pm 0.3^{*,+}$ & $17.5 \pm 0.8$ & $0.7 \pm 0.1^{+}$ & $0.6 \pm 0.3$ & $22.3 \pm 1.2$ & $0.5 \pm 0.1$ & $3.0 \pm 0.6$ \\
\hline High fat+menhaden oil (8) & $22.5 \pm 0.7^{+}$ & $19.9 \pm 0.5$ & $6.8 \pm 0.4$ & $14.4 \pm 0.5$ & ND & $0.4 \pm 0.1$ & $11.9 \pm 0.4^{*,+}$ & $4.4 \pm 0.4^{*,+}$ & $7.6 \pm 0.6^{*,+}$ \\
\hline
\end{tabular}

Notes: Data are presented as the mean \pm standard error of mean. ${ }^{*} p<0.05$ compared to control; ${ }^{+} p<0.05$ compared to high fat. The number of animals in each group is shown in parenthesis.

Abbreviation: ND, not detected. 
Table S3 Effect of dietary lipids on fatty acid \% composition of liver measured by gas chromatography

\begin{tabular}{llllllllll}
\hline Diet & $16: 0$ & $18: 0$ & $18: 1$ & $18: 2$ & $18: 3(\mathrm{n}-6)$ & $18: 3(\mathrm{n}-3)$ & $20: 4$ & $20: 5$ & $22: 6$ \\
Control (9) & $17.1 \pm 4.3$ & $15.4 \pm 1.9$ & $12.7 \pm 2.0$ & $11.9 \pm 0.5$ & $0.3 \pm 0.1$ & $0.2 \pm 0.1$ & $21.6 \pm 2.3$ & $0.2 \pm 0.1$ & $6.8 \pm 0.3$ \\
High fat (10) & $18.3 \pm 0.3$ & $19.0 \pm 1.3$ & $12.9 \pm 15.0$ & $15.0 \pm 0.8$ & $0.4 \pm 0.1$ & $0.4 \pm 0.1$ & $20.4 \pm 1.1$ & $0.3 \pm 0.1$ & $5.5 \pm 0.4$ \\
High fat+olive oil (9) & $14.6 \pm 2.4$ & $19.5 \pm 0.7$ & $14.9 \pm 1.6$ & $12.1 \pm 1.0$ & $0.3 \pm 0.1$ & $0.2 \pm 0.1$ & $23.8 \pm 0.4$ & $0.2 \pm 0.1$ & $6.9 \pm 0.5$ \\
High fat+safflower oil (9) & $15.3 \pm 0.7$ & $18.7 \pm 0.5$ & $8.9 \pm 0.4$ & $19.0 \pm 1.3^{*,+}$ & $0.7 \pm 0.2$ & $0.3 \pm 0.1$ & $23.3 \pm 1.1^{\prime}$ & $0.3 \pm 0.1$ & $5.2 \pm 0.3$ \\
High fat+flaxseed oil (9) & $17.8 \pm 0.3$ & $16.2 \pm 2.0$ & $11.1 \pm 0.8$ & $15.0 \pm 0.4$ & $0.3 \pm 0.1$ & $4.3 \pm 0.6^{*,+}$ & $14.8 \pm I .1^{*,+}$ & $3.5 \pm 0.6^{*,+}$ & $7.0 \pm 0.7$ \\
High fat+evening primrose oil (8) & $15.8 \pm 0.7$ & $20.3 \pm 0.7^{*}$ & $7.2 \pm 0.4$ & $17.4 \pm 1.0^{*}$ & $0.7 \pm 0.1$ & $0.5 \pm 0.2$ & $26.0 \pm 0.7^{+}$ & $0.4 \pm 0.2$ & $4.1 \pm 0.2^{*}$ \\
High fat+menhaden oil (9) & $18.7 \pm 0.9$ & $17.4 \pm 0.6$ & $8.4 \pm 0.6$ & $13.6 \pm 0.3$ & $0.3 \pm 0.2$ & $0.5 \pm 0.1$ & $14.3 \pm 0.8^{*,+}$ & $5.2 \pm 0.5^{*,+}$ & $13.0 \pm 0.4^{*++}$ \\
\hline
\end{tabular}

Notes: Data are presented as the mean \pm standard error of mean. ${ }^{*} p<0.05$ compared to control; ${ }^{*} p<0.05$ compared to high fat. The number of animals in each group is shown in parenthesis.

Table S4 Fatty acid unsaturation indices of liver and serum measured by gas chromatography

\begin{tabular}{lll}
\hline Diet & Liver & Serum \\
\hline Control & $1.75 \pm 0.06$ & $1.51 \pm 0.08$ \\
High fat & $1.71 \pm 0.04$ & $1.56 \pm 0.04$ \\
High fat+olive oil & $1.87 \pm 0.03^{+}$ & $1.63 \pm 0.05$ \\
High fat+safflower oil & $1.83 \pm 0.03$ & $1.59 \pm 0.03$ \\
High fat+evening primrose oil & $1.85 \pm 0.02$ & $1.63 \pm 0.04$ \\
High fat+flaxseed oil & $1.87 \pm 0.04^{+}$ & $1.62 \pm 0.04$ \\
High fat+menhaden oil & $2.16 \pm 0.03^{*+}$ & $1.65 \pm 0.05$ \\
\hline
\end{tabular}

Notes: Data are presented as the mean \pm standard error of mean. ${ }^{*} p<0.05$ compared to control; ${ }^{+} p<0.05$ compared to high fat.

Table S5 Ratio of n-6 to n-3 fatty acids of liver and serum measured by gas chromatography

\begin{tabular}{lll}
\hline Diet & Liver & Serum \\
\hline Control & $4.39 \pm 0.27$ & $7.02 \pm 0.7 \mathrm{I}$ \\
High fat & $5.07 \pm 0.23$ & $8.35 \pm 0.74$ \\
High fat+olive oil & $4.49 \pm 0.37$ & $6.27 \pm 0.78$ \\
High fat+safflower oil & $6.80 \pm 0.48^{*,+}$ & $9.49 \pm 1.15$ \\
High fat+evening primrose oil & $8.09 \pm 0.45^{*,+}$ & $9.60 \pm 1.38$ \\
High fat+flaxseed oil & $1.87 \pm 0.11^{*,+}$ & $3.40 \pm 0.38^{*,+}$ \\
High fat+menhaden oil & $1.39 \pm 0.10^{*,+}$ & $1.96 \pm 0.11^{*,+}$ \\
\hline
\end{tabular}

Notes: Data are presented as the mean \pm standard error of mean. ${ }^{*} p<0.05$ compared to control; ${ }^{+} p<0.05$ compared to high fat.

opinion and commentaries are all considered for publication. The manuscript management system is completely online and includes a very quick and fair peer-review system, which is all easy to use. Visit http://www.dovepress.com/testimonials.php to read real quotes from published authors.

Submit your manuscript here: https://www.dovepress.com/diabetes-metabolic-syndrome-and-obesity-targets-and-therapy-journal 